Hemangioma cráneo-facio-cervical y subglótico con clínica respiratoria. Una urgencia terapéutica

\title{
Cranio-facial-cervical and subglottic hemangioma with respiratory symptoms. A therapeutic emergency
}

https://doi.org/10.23938/ASSN.0929

\author{
J.C. Moreno Alfonso ${ }^{1}$, A.Y. Molina Caballero' ${ }^{1}$, A. Pérez Martínez ${ }^{1}$, \\ J. Arredondo Montero' ${ }^{1}$, J. Zubicaray Ugarteche ${ }^{2}$, C. Bardají Pascual ${ }^{1}$
}

\section{RESUMEN}

Se describe el caso de una lactante de 41 días de vida con un hemangioma cráneo-facio-cervical izquierdo y subglótico con repercusión respiratoria. Aunque los hemangiomas infantiles son tumores vasculares benignos que se presentan predominantemente en piel y de forma aislada, un 1-2\% de los pacientes pueden tener lesiones en la vía aérea que ocasionalmente provocan cuadros respiratorios potencialmente mortales.

Se decidió iniciar inmediatamente el tratamiento con propranolol, sin esperar a completar el diagnóstico de extensión y sindrómico, con buena respuesta clínica y desaparición de la sintomatología respiratoria en las siguientes doce horas. En los niños con hemangiomas que por su localización produzcan síntomas o alteración funcional importante, es imprescindible iniciar el tratamiento betabloqueante de forma precoz y en algunos casos, urgentemente.

Palabras clave: Hemangioma subglótico. Propranolol. Estridor.

\begin{abstract}
We describe the case of a 41-day-old infant with a left craniofacial cervical and subglottic hemangioma with respiratory symptoms. Although infantile hemangiomas are occasional benign vascular tumors that appear predominantly on the skin, $1-2 \%$ of patients may have airway lesions that can sometimes cause potentially life-threatening respiratory conditions.

The decision was made to immediately commence treatment with propranolol, without waiting to complete the extension and syndromic diagnoses. There was a positive clinical response and respiratory symptoms dissipated in twelve hours. Early treatment with beta-blockers is essential for children with hemangiomas whose location causes symptoms or significant functional changes: in some cases it may be a matter of urgency.
\end{abstract}

Keywords: Subglottic hemangioma. Propranolol. Stridor.
1. Cirugía Pediátrica. Complejo Hospitalario de Navarra. Pamplona. España.

2. Otorrinolaringología Pediátrica. Complejo Hospitalario de Navarra. Pamplona. España.

Recepción: 30/09/2020

Aceptación provisional: 23/10/2020

Aceptación definitiva: 07/11/2020

\section{Correspondencia:}

Julio César Moreno Alfonso

Servicio de Cirugía Pediátrica

Complejo Hospitalario de Navarra

C/ Irunlarrea, 3

31008 Pamplona

E-mail: jc.moreno.alfonso@navarra.es 


\section{INTRODUCCIÓN}

Los hemangiomas infantiles (HI) son los tumores vasculares benignos más frecuentes de la infancia (4-5\% de la población infantil), producidos por una rápida proliferación de las células endoteliales de los vasos sanguíneos ${ }^{1-3}$. Se caracterizan por una fase de proliferación en el primer año de vida seguida de una fase de involución en la que el componente vascular es reemplazado por tejido fibroadiposo. La mayoría no son clínicamente evidentes al nacimiento, sino que aparecen a lo largo de los primeros días o semanas de vida, aunque algunos pacientes pueden presentar al nacer una lesión cutánea precursora a modo de telangiectasia o mácula rosada. Tienen predilección por la cabeza y el cuello, pero pueden asentarse en cualquier parte del cuerpo, incluyendo mucosas y órganos internos, y medir desde unos pocos milímetros hasta varios centímetros de diámetro ${ }^{3-6}$. Generalmente se presentan como lesiones cutáneas aisladas, si bien algunos forman parte de diversos síndromes o malformaciones, y en el $1-2 \%$ de los pacientes con HI cutáneos existen lesiones asociadas en la vía aérea que pueden ocasionar cuadros clínicos graves e incluso fatales $^{4,7-9}$.

La mayoría de los HI no necesitan tratamiento. En los casos indicados, el propranolol se considera la primera línea terapéutica. Este fármaco es un betabloqueante no selectivo que inhibe el crecimiento e induce la regresión de los $\mathrm{HI}$ gracias a su efecto vasoconstrictor, disminución de la expresión de factores de crecimiento endotelial vascular y fibroblástico e inducción de la apoptosis ${ }^{5}$. El propranolol ha revolucionado el tratamiento de los $\mathrm{HI}$ ya que es un medicamento con pocos efectos secundarios y con un alto índice de respuesta $^{5,6,10}$.

Se presenta el caso de una paciente de 41 días de vida con un hemangioma cráneofacio-cervical izquierdo y subglótico con repercusión respiratoria, en el que se planteó la duda entre completar el diagnóstico de extensión y sindrómico demorando el inicio del tratamiento, o comenzar la administración de propranolol sin retrasos. Este artículo analiza las implicaciones de ambas conductas clínicas y por qué se optó por la segunda alternativa.

\section{CASO CLÍNICO}

Lactante diagnosticada al nacimiento de un hemangioma segmentario hemifacial y craneocervical en región auricular izquierda que progresivamente, en los siguientes días, se fue extendiendo hacia región témporo-occipital y laterocervical ipsilateral con lesiones costrosas y ulceradas, afectando a la audición del oído izquierdo (estudio de otoemisiones acústicas alterado).

A los 41 días de vida la paciente ingresa por un cuadro clínico de seis días de evolución consistente en disfonía y dificultad respiratoria refractaria a manejo corticoide, que empeoraba con el decúbito y el llanto y mejoraba con la sedestación. Al nacer había presentado un episodio similar que requirió ingreso durante cinco días, y otro a los once días de vida que fue manejado de manera ambulatoria.

En el examen físico se apreciaba el hemangioma cutáneo ya conocido (Fig. 1), con auscultación cardíaca, pulmonar y resto de exploraciones normales. Se le realizó, por parte del servicio de Otorrinolaringología (ORL), una nasofibrolaringoscopia en la que se observaba una tumoración subglótica de extirpe vascular, motivo por el que se consultó a Cirugía Pediátrica. En ese momento se valoró la posibilidad de realizar una fibrotraqueoscopia y resonancia magnética (RM) craneal y de tórax para establecer la extensión y presencia de otras lesiones vasculares o malformaciones asociadas a síndromes vasculares que pudieran condicionar el tratamiento. Ante la afectación de la vía aérea con clínica respiratoria grave, se inició inmediatamente el tratamiento con propranolol previa valoración por Cardiología Pediátrica para descartar malformaciones cardiacas y alteraciones del ritmo que contraindicaran el tratamiento con betabloqueantes. La dosis inicial fue de $2 \mathrm{mg} / \mathrm{kg} /$ día por vía oral cada 12 horas, con muy buena respuesta. No se presentaron complicaciones, los síntomas respiratorios desaparecieron tras la segunda dosis y fue dada de alta dos días después.

El tratamiento se mantuvo durante once meses con dosis ascendentes ajustadas a la ganancia ponderal, sin efectos secundarios, con gran mejoría de la lesión cutánea desde la segunda semana (Fig. 2), desaparición de la clínica respiratoria y del hemangioma subglótico (comprobado por ORL a los dos meses de vida) y normaliza- 


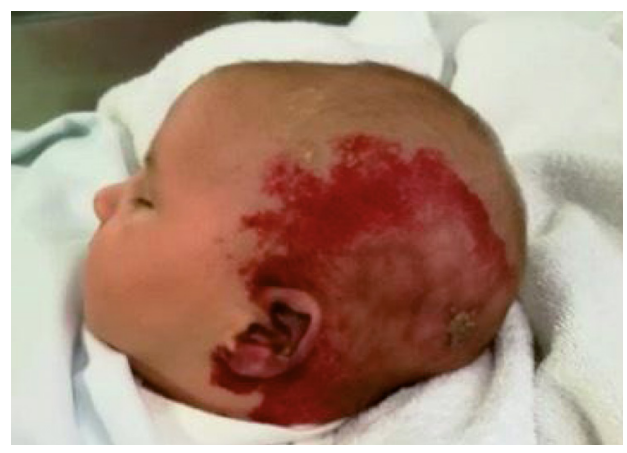

Figura 1. Hemangioma cráneo-facio-cervical antes de la administración del propranolol.

ción de la función auditiva del oído izquierdo. Se completó el estudio con ecografía de abdomen y RM craneal y torácica a los dos y siete meses, respectivamente, que descartaron la existencia de lesiones traqueales o anomalías asociadas. Después de un año de seguimiento tras la suspensión del tratamiento, no ha habido recidiva de la afectación cutánea ni de la clínica respiratoria.

\section{DISCUSIÓN}

La localización subglótica de los HI es rara, representando el 1,5\% de todas las lesiones laríngeas congénitas ${ }^{4,10}$, pero deben ser considerados en cualquier niño con un hemangioma cervicofacial o mandibular y desarrollo progresivo de sibilancias y estridor, síntoma más frecuente de los subglóticos, usualmente bifásico y de predominio inspiratorio ${ }^{2,4,7}$.

Los niños afectados tienen mayor probabilidad de experimentar síntomas entre las seis y las doce semanas, cuando la proliferación del hemangioma es más rápida?. Estos síntomas pueden progresar desde tos, sibilancias, cianosis y estridor hasta la insuficiencia respiratoria. En 2009, Denoyelle y col publicaron los primeros casos de HI subglóticos tratados satisfactoriamente con propranolol ${ }^{11}$. Desde entonces, el propranolol se ha convertido en el estándar de tratamiento de estas lesiones, evitando los efectos adversos asociados al uso prolongado de corticoides, las complicaciones de la cirugía abierta y la traqueotomía ${ }^{12-14}$.

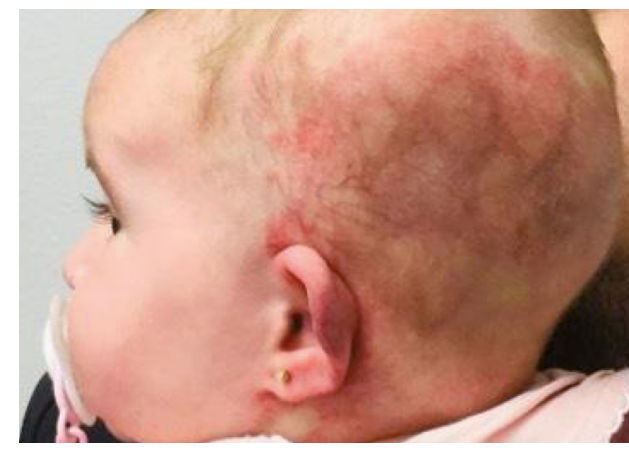

Figura 2. Hemangioma cráneo-facio-cervical once meses después del tratamiento con propranolol.

Independientemente de su baja incidencia, los HI subglóticos son una entidad grave y potencialmente mortal debido a que el grado de obstrucción supera el $70 \%$ de la luz traqueal en dos tercios de los casos, con una tasa de mortalidad cercana al 50\% si no se $\operatorname{tratan}^{13,14}$. Teniendo en cuenta la rápida respuesta al propranolol observada en los pacientes con HI subglóticos, con desaparición del estridor en las primeras 24 horas o menos de iniciado el tratamiento ${ }^{13,15}$ y el potencial riesgo vital asociado a la edad de la paciente, la proliferación de las lesiones vasculares y la sintomatología respiratoria grave refractaria a tratamiento con corticoides, se inició de forma inmediata el tratamiento con propranolol oral antes de completar el estudio por imagen. Estuvo latente, ante la presencia de un hemangioma facial grande y segmentario, la posibilidad de estar frente a un Síndrome de PHACE. Este es el acrónimo en inglés utilizado para describir la asociación de un hemangioma cervicofacial grande, con malformaciones de la fosa posterior cerebral, displasia de arterias cerebrales, defectos cardíacos o coartación aórtica y anomalías oculares ${ }^{7}$. Clásicamente se ha cuestionado la seguridad del propranolol en estos pacientes ya que el uso de $\beta$-bloqueantes en el contexto de la vasculopatía cerebral podría, teóricamente, aumentar el riesgo de un episodio isquémico cerebral. Sin embargo, recientemente Olsen y col publicaron un estudio multicéntrico retrospectivo con 76 
pacientes con síndrome de PHACE tratados con propranolol vía oral sin encontrar diferencias significativas en la tasa de eventos adversos severos (ictus, accidentes cardiovasculares e isquémicos transitorios) respecto a aquellos que solo tenían la lesión vascular cutánea ( $0 \%$ vs $0,4 \%$, respectivamente) ${ }^{16}$.

Todo lo anterior respaldó la decisión de anteponer el inicio del tratamiento frente a demorarlo realizando otros estudios de extensión para descartar malformaciones asociadas. Se desistió de la fibrotraqueoscopia para caracterizar las lesiones de la vía aérea por el riesgo de provocar un sangrado o un broncoespasmo grave durante la exploración. Como se ha visto reflejado en este caso, la indicación de tratamiento en los $\mathrm{HI}$ debe ser individualizada, basada en las características propias de la lesión y la presencia de complicaciones o afectación funcional grave. Es importante recordar que un diagnóstico y tratamiento precoz mejora el pronóstico de estos pacientes $\mathrm{y}$ reduce las secuelas.

En conclusión, en aquellos niños con $\mathrm{HI}$ que por su localización produzcan una clínica o alteración funcional importante, es imprescindible iniciar el tratamiento con propranolol de forma precoz y en algunos casos, incluso de forma urgente, sin esperar a completar el estudio diagnóstico. En este caso, el riesgo de complicaciones graves asociadas al propranolol era muy bajo y asumible ante la gravedad del cuadro respiratorio.

\section{BIBLIOGRAFÍA}

1. Baselga E, Dembowska B, Przewratil P, Gonzalez MA, WyrzyKowsKi D, TORRElo A et al. Efficacy of propranolol between 6 and 12 months of age in high risk infantile hemangioma. Pediatrics 2018; 142: e20173866. https://doi. org/10.1542/peds.2017-3866

2. Solman L, Glover M, Beattle PE, Buckley $\mathrm{H}$, GACH JE, Giardini A et al. Oral propranolol in the treatment of proliferating infantile haemangiomas: The British Society for Paediatric Dermatology consensus guidelines. $\mathrm{Br}$ J Dermatol 2018; 179: 582-589. https://doi. org/10.1111/bjd.16779
3. Metry DW. Infantile hemangiomas: Epidemiology, pathogenesis, clinical features, and complications. En: Levy ML, Corona R, editores. Waltham: UpToDate 2019. https://www. uptodate.com/contents/infantile-hemangiomas-epidemiology-pathogenesis-clinical-features-and-complications/print

4. Mendiratta V, Varghese B, Chander R, Parakh A, SolANKI R. Successful management of airway hemangioma with propranolol. Int J Dermatol 2013; 52: 750-752. https://doi.org/10.1111/ j.1365-4632.2012.05752.x

5. Laranjo S, Costa G, Parames F, Freitas i, Diogo $\mathrm{J}$, TRIGo $\mathrm{C}$ et al. The role of propranolol in the treatment of infantile hemangioma. Rev Port Cardiol 2014; 33: 289-295. https://doi. org/10.1016/j.repc.2013.10.018

6. Cheirif O, Novelo AD, Orozco L, SaEz M. Hemangioma infantil: actualización del tratamiento tópico y sistémico. Bol Med Hosp Infant Mex 2019; 76: 167-175. https://doi.org/10.24875/ bmhim. 19000002

7. Vivas GV, Fernandez I, Lopez JC, Fernandez MA, Garcia MA, Matute de Cardenas JA. Analysis of the therapeutic evolution in the management of airway infantile hemangioma. World J Clin Pediatr 2016; 5: 95-101. https://doi. org/10.5409/wjcp.v5.i1.95

8. Igari Y, Takahashi S, Usui A, Kanasumi E, Funayama M. Sudden death in a child caused by a giant cavernous hemangioma of the anterior mediastinum. J Forensic Leg Med 2017; 52: 9397. https://doi.org/10.1016/j.jflm.2017.08.008

9. Littler ER, Arbor A. Asphyxia due to hemangioma in trachea. J Thorac Cardiovasc Surg 1963; 45: 552-558.

10. Hoeger PH, Harper Ji, Baselga E, Bonnet D, Boon LM, Degli MC et al. Treatment of infantile haemangiomas: Recommendations of a European expert group. Eur J Pediatr 2015; 174: 855-865. https://doi.org/10.1007/s00431015-2570-0

11. Denoyelle F, Leboulanger N, Enjolras O, Harris R, Roger G, GARABEDIAN EN. Role of propranolol in the therapeutic strategy of infantile laryngotracheal hemangioma. Int J Pediatr Otorhinolaryngol 2009; 73: 1168-1172. https://doi. org/10.1016/j.ijporl.2009.04.025

12. Onder S, Gergin O, Karabulut B. A life threatening subglottic and mediastinal hemangioma in an infant. J Craniofac Surg 2019; 30: e402-e404. https://doi.org/10.1097/ scs.0000000000005340

13. Álvarez H, Penchyna J, Teyssier G, Morera E, De la Torre C, Villamor P. Tiempo de respuesta terapéutica al propranolol sistémico en el manejo de hemangiomas subgló- 
ticos pediátricos: serie de casos y revisión de la literatura. Bol Med Hosp Infant Mex 2018; 75: 377-382. https://doi.org/10.24875/ bmhim. 18000043

14. Torrelo A, Tamariz A, Domínguez MJ. Observaciones clínicas sobre el uso de propranolol en hemangiomas de vía aérea infantil. Acta Otorrinolaringol Esp 2010; 61: 365-370. https://doi.org/10.1016/j.otorri.2010.01.014

15. Elluru RG, Friess MR, Richter GT, Grimmer JF, DARrow DH, SHIN JJ et al. Multicenter evaluation of the effectiveness of systemic propranolol in the treatment of airway hemangiomas. Otolaryngol Head Neck Surg 2015; 153: 452-460. https://doi. org/10.1177/0194599815591809

16. Olsen GM, Hansen LM, Stefanko NS, Mathes E, Puttgen KB, Tollefson MM et al. Evaluating the safety of oral propranolol therapy in patients with PHACE Syndrome. JAMA Dermatol 2020; 156: 186-190. https://doi. org/10.1001/jamadermatol.2019.3839 
